\title{
Stability of traveling wavefronts for a 2D lattice dynamical system arising in a diffusive population model
}

Haiqin Zhao ${ }^{1 *}$

\section{"Correspondence:}

zhaohaiqin@xidian.edu.cn

'School of Mathematics and

Statistics, Xidian University, Xi'an,

People's Republic of China

\section{Springer}

\begin{abstract}
This paper is concerned with the traveling wavefronts of a 2D two-component lattice dynamical system. This problem arises in the modeling of a species with mobile and stationary subpopulations in an environment in which the habitat is two-dimensional and divided into countable niches. The existence and uniqueness of the traveling wavefronts of this system have been studied in (Zhao and Wu in Nonlinear Anal., Real World Appl. 12: 1178-1191, 2011). However, the stability of the traveling wavefronts remains unsolved. In this paper, we show that all noncritical traveling wavefronts with given direction of propagation and wave speed are exponentially stable in time. In particular, we obtain the exponential convergence rate.
\end{abstract}

MSC: 35K57; 35R10; 35B40; 92D25

Keywords: Lattice differential system; Traveling wavefronts; Quiescent stage

\section{Introduction}

In this paper, we consider the following two-dimensional (2D) lattice differential system:

$$
\left\{\begin{array}{l}
\frac{\mathrm{d} u_{i, j}(t)}{\mathrm{d} t}=\left(\Delta_{2} u\right)_{i, j}+f\left(u_{i, j}(t)\right)-\gamma_{1} u_{i, j}(t)+\gamma_{2} v_{i, j}(t), \\
\frac{\mathrm{d} v_{i, j}(t)}{\mathrm{d} t}=\gamma_{1} u_{i, j}(t)-\gamma_{2} v_{i, j}(t),
\end{array}\right.
$$

where $u_{i, j}(t) \in \mathbb{R}, v_{i, j}(t) \in \mathbb{R}, i, j \in \mathbb{Z}, t>0$, and

$$
\left(\Delta_{2} u\right)_{i, j}:=d\left[u_{i+1, j}+u_{i-1, j}+u_{i, j+1}+u_{i, j-1}-4 u_{i, j}\right] .
$$

The system models a species with mobile and stationary subpopulations in an environment in which the habitat is two-dimensional and divided into countable niches (see [4]). Such behavior is typical for invertebrates living in small ponds in arid climates, which dry up and reappear subject to rainfall (Hadeler and Lewis [4]). Here $u_{i, j}(t)$ and $v_{i, j}(t)$ represent the densities of mobile and stationary subpopulations at a point $(i, j)$ and time $t$, respectively, $d>0$ is the diffusion coefficient of the mobile subpopulation, and $f$ is the reproduction function. The rates of transfer between two states are assumed to be $\gamma_{1}>0$ and $\gamma_{2}>0$.

(c) The Author(s) 2019. This article is distributed under the terms of the Creative Commons Attribution 4.0 International License (http://creativecommons.org/licenses/by/4.0/), which permits unrestricted use, distribution, and reproduction in any medium, provided you give appropriate credit to the original author(s) and the source, provide a link to the Creative Commons license, and indicate if changes were made. 
In fact, system (1.1) is a spatially discrete version of the following continuous model with a quiescent stage (see Hadeler and Lewis [4]):

$$
\left\{\begin{array}{l}
\partial_{t} u(x, t)=d \Delta u(x, t)+f(u(x, t))-\gamma_{1} u(x, t)+\gamma_{2} v(x, t), \\
\partial_{t} v(x, t)=\gamma_{1} u(x, t)-\gamma_{2} v(x, t) .
\end{array}\right.
$$

However, in some cases, a lattice differential model is more suitable than the continuous one. For example, the ponds, the living environment of frogs, which are scattered in the grassland, cannot be regarded as a continuous environment. Actually, lattice dynamical systems have been extensively used to model biological and epidemic problems, and some new phenomenon has been observed in discrete models, such as occurrence of "propagation failure" or "pinning"; we refer to the survey paper [3] and book [14].

The traveling wave and related problems of systems (1.1) and (1.2) have been widely studied. Zhang and Zhao [20] established the existence of spreading speed of (1.2) and showed that it coincides with the minimal wave speed for traveling wavefronts. Zhang and $\mathrm{Li}$ [21] further considered the monotonicity and uniqueness of the traveling wave solutions of (1.2). Recently, Zhao and Wu [22] studied the existence, asymptotic behavior, monotonicity, and uniqueness of traveling wavefronts of the 2D discrete model (1.1). The effects of the direction of propagation on the minimal wave speed $c_{*}(\theta)$ is also investigated. We found that: (i) $c_{*}(\theta)$ is a periodic function with period $\frac{\pi}{2}$ and has the symmetry in $\theta=\frac{\pi}{4}$; (ii) the $1 \mathrm{D}$ front (i.e., $\left.\theta=0, \frac{\pi}{2}\right)$ is the fastest, and the diagonal front (i.e., $\theta=\frac{\pi}{4}$ ) is the slowest; (iii) $c_{*}(\theta)$ is monotonically decreasing and increasing in $\theta \in\left[0, \frac{\pi}{4}\right]$ and $\theta \in\left[\frac{\pi}{4}, \frac{\pi}{2}\right]$, respectively. However, the stability of the traveling wavefronts for systems (1.1) and (1.2) remains unsolved. This constitutes the purpose of this paper.

In the past decades, there are many techniques developed to deal with the stability of the traveling waves (see $[1,2,5,6,9-13,16,17,19])$, such as the spectral analysis method ([1, $19])$, the weighted-energy method ([12]), the sub- and supersolutions combining squeezing technique $([2,17])$, and the combination of the comparison principle and the weightedenergy method $([9,11])$. More recently, Ouyang and $\mathrm{Ou}[13]$ used a new method to prove the asymptotic stability of traveling waves of a nonlocal reaction-diffusion equation in periodic media. In this paper, we extend this method to systems (1.1) and (1.2). We focus on the stability of the traveling wavefronts for $2 D$ discrete system (1.1), since the same issue for the continuous model (1.2) can be treated similarly. More precisely, by establishing a comparison theorem for a related initial-boundary value problem (see Lemma 3.1) and giving a variational characterization of the minimal wave speed (see Lemma 2.4) we show that any traveling wavefront of (1.1) with given direction of propagation $\theta \in[0,2 \pi]$ and wave speed $c>c_{*}(\theta)$ is exponentially stable in time (see Theorem 3.2). In particular, we obtain the exponential convergence rate.

Throughout this paper, we make the following assumptions on the function $f$ :

$\left(A_{1}\right) f \in C^{2}[0,1], f(0)=f(1)=0, f^{\prime}(0)>0, f^{\prime}(1)<0$, and $f(u)>0$ for all $u \in(0,1)$.

$\left(A_{2}\right) f^{\prime}(u) \leq f^{\prime}(0)$ for all $u \in[0,1]$.

From $\left(A_{1}\right)$ we see that $(1.1)$ has two equilibria $\mathbf{0}:=(0,0)$ and $\mathbf{K}:=(1, K)$, where $K:=\gamma_{1} / \gamma_{2}$. We are interested in traveling wave solutions that connect the two equilibria $\mathbf{0}$ and $\mathbf{K}$. A solution $w_{i, j}(t)=\left(u_{i, j}(t), v_{i, j}(t)\right)$ of (1.1) is called a traveling wave solution of (1.1) with speed $c$ and direction $\theta$ if there exist constants $\theta \in \mathbb{R}$ and $c>0$ and a differentiable function 
$W=(U, V): \mathbb{R} \rightarrow \mathbb{R}^{2}$ such that

$$
\left(u_{i, j}(t), v_{i, j}(t)\right)=(U(i \cos \theta+j \sin \theta+c t), V(i \cos \theta+j \sin \theta+c t)), \quad \forall i, j \in \mathbb{Z}, t \in \mathbb{R} .
$$

Moreover, if $W: \mathbb{R} \rightarrow \mathbb{R}^{2}$ is monotone, then we say that $W$ is a traveling wavefront.

The rest of this paper is organized as follows. In Sect. 2, we first recall a result on the existence of the minimal wave speed $c_{*}(\theta)$. Then we give a variational characterization of $c_{*}(\theta)$. Section 3 is devoted to the stability of noncritical traveling wavefronts.

\section{Characterization of minimal wave speed}

In this section, we first recall some results on the existence of the minimal wave speed $c_{*}(\theta)$. Then we give a variational characterization of $c_{*}(\theta)$, which will be used in proving our stability result.

For $c \geq 0$ and $\lambda \in \mathbb{C}$ with $\lambda \neq-\frac{\gamma_{2}}{c}$, we define the characteristic function as follows:

$$
\triangle(c, \lambda)=c \lambda-d\left[e^{\lambda \cos \theta}+e^{-\lambda \cos \theta}+e^{\lambda \sin \theta}+e^{-\lambda \sin \theta}-4\right]-f^{\prime}(0)+\gamma_{1}-\frac{\gamma_{1} \gamma_{2}}{c \lambda+\gamma_{2}} .
$$

We have the following results on the existence of the minimal wave speed.

Lemma 2.1 ([22, Lemma 2.5]) For any fixed $\theta \in \mathbb{R}$, there exists a positive number $c_{*}(\theta)$ such that

(i) if $c \geq c_{*}(\theta)$, then $\triangle(c, \lambda)=0$ has two roots $\lambda_{1}(c), \lambda_{2}(c)$ with $0<\lambda_{1}(c) \leq \lambda_{2}(c)$;

(ii) if $c=c_{*}(\theta)$, then $\lambda_{1}\left(c_{*}\right)=\lambda_{2}\left(c_{*}\right):=\lambda_{*}(\theta)$, and if $c>c_{*}(\theta)$, then $\lambda_{1}(c)<\lambda_{*}(\theta)<\lambda_{2}(c)$, and $\triangle(c, \cdot)<0$ in $\mathbb{R} \backslash\left(\lambda_{1}(c), \lambda_{2}(c)\right), \Delta(c, \cdot)>0$ in $\left(\lambda_{1}(c), \lambda_{2}(c)\right)$.

Lemma 2.2 ([22, Theorem 2.7]) Let $\theta \in \mathbb{R}$. Then:

(i) for each $c \geq c_{*}(\theta)$, (1.1) has a traveling wavefront $W(\xi), \xi=i \cos \theta+j \sin \theta+c t$, with direction of propagation $\theta$ and wave speed $c$;

(ii) for any $0<c<c_{*}(\theta)$, (1.1) has no traveling wave solutions.

Now, we give a variational characterization of $c_{*}(\theta)$. Substituting

$$
\left(u_{i, j}(t), v_{i, j}(t)\right)=\mathrm{e}^{\lambda(i \cos \theta+j \sin \theta)}(u(t), v(t)), \quad \lambda \in \mathbb{R},
$$

into the linearized system of $(1.1)$ at $(0,0)$, we obtain

$$
\left\{\begin{array}{l}
u^{\prime}(t)=d\left[e^{\lambda \cos \theta}+e^{-\lambda \cos \theta}+e^{\lambda \sin \theta}+e^{-\lambda \sin \theta}-4\right] u(t)+\left[f^{\prime}(0)-\gamma_{1}\right] u(t)+\gamma_{2} v(t), \\
v^{\prime}(t)=\gamma_{1} u(t)-\gamma_{2} v(t) .
\end{array}\right.
$$

Clearly, the eigenvalue problem of this system has the following from:

$$
\left\{\begin{array}{l}
M(\lambda) v_{1}=d\left[e^{\lambda \cos \theta}+e^{-\lambda \cos \theta}+e^{\lambda \sin \theta}+e^{-\lambda \sin \theta}-4\right] v_{1}+\left[f^{\prime}(0)-\gamma_{1}\right] v_{1}+\gamma_{2} \nu_{2}, \\
M(\lambda) v_{2}=\gamma_{1} v_{1}-\gamma_{2} \nu_{2} .
\end{array}\right.
$$

From [15, Theorem 5.5.1], we see that problem (2.1) has a principal eigenvalue $M(\lambda)$ with a strictly positive eigenfunction $v(\lambda)=\left(v_{1}(\lambda), v_{2}(\lambda)\right)$. 
Lemma 2.3 Let $\Phi(\lambda)=M(\lambda) / \lambda$. Then:

(i) $\lim _{\lambda \rightarrow 0+} \Phi(\lambda) \rightarrow \infty$ and $\lim _{\lambda \rightarrow \infty} \Phi(\lambda)=\infty$.

(ii) $\Phi(\lambda)$ is decreasing near 0 , and $\Phi^{\prime}(\lambda)$ changes the sign at most once on $(0, \infty)$.

(iii) There exists $\lambda^{*}=\lambda^{*}(\theta) \in(0, \infty)$ such that $c^{*}(\theta):=\Phi\left(\lambda^{*}\right)=\inf _{\lambda>0} \Phi(\lambda)$.

Proof It is clear that $M(\lambda)$ is the spectral radius of a matrix and

$$
\begin{aligned}
M(\lambda) & =\frac{H(\lambda)+\sqrt{H^{2}(\lambda)+4\left\{d\left[e^{\lambda \cos \theta}+e^{-\lambda \cos \theta}+e^{\lambda \sin \theta}+e^{-\lambda \sin \theta}-4\right]+f^{\prime}(0)\right\} \gamma_{2}}}{2} \\
& \geq \sqrt{f^{\prime}(0) \gamma_{2}},
\end{aligned}
$$

where

$$
H(\lambda)=d\left[e^{\lambda \cos \theta}+e^{-\lambda \cos \theta}+e^{\lambda \sin \theta}+e^{-\lambda \sin \theta}-4\right]+f^{\prime}(0)-\gamma_{1}-\gamma_{2} .
$$

Hence $\lim _{\lambda \rightarrow 0+} \Phi(\lambda) \rightarrow \infty$. Moreover, by the first equation of (2.1) we get

$$
\Phi(\lambda) \geq \frac{d\left[e^{\lambda \cos \theta}+e^{-\lambda \cos \theta}+e^{\lambda \sin \theta}+e^{-\lambda \sin \theta}-4\right]}{\lambda}+\frac{f^{\prime}(0)-\gamma_{1}}{\lambda} .
$$

Since $\cos ^{2} \theta+\sin ^{2} \theta=1$, it is clear that $\lim _{\lambda \rightarrow \infty} \Phi(\lambda)=\infty$. Then the lemma directly follows from Liang and Zhao [7, Lemma 3.8].

The following result says that $c^{*}(\theta)$ equals the minimal wave speed $c_{*}(\theta)$.

Lemma 2.4 For any given $\theta \in \mathbb{R},\left(c^{*}(\theta), \lambda^{*}(\theta)\right)=\left(c_{*}(\theta), \lambda_{*}(\theta)\right)$.

Proof For convenience, we denote $\left(c^{*}(\theta), \lambda^{*}(\theta)\right)$ and $\left(c_{*}(\theta), \lambda_{*}(\theta)\right)$ by $\left(c^{*}, \lambda^{*}\right)$ and $\left(c_{*}, \lambda_{*}\right)$, respectively. By Lemma 2.1 we see that $\left(c_{*}, \lambda_{*}\right)$ is a unique solution of the system

$$
\triangle(c, \lambda)=0 \quad \text { and } \quad \frac{\partial}{\partial \lambda} \triangle(c, \lambda)=0 .
$$

From Lemma 2.3 we have $\Phi\left(\lambda^{*}\right)=M\left(\lambda^{*}\right) / \lambda^{*}=c^{*}$ and $\Phi^{\prime}\left(\lambda^{*}\right)=0$. Hence

$$
M\left(\lambda^{*}\right)=c^{*} \lambda^{*} \quad \text { and } \quad M^{\prime}\left(\lambda^{*}\right)=\frac{M\left(\lambda^{*}\right)}{\lambda^{*}}=c^{*} .
$$

Letting $v_{1}=1$, it then follows from (2.1) that $v_{2}=\gamma_{1} /\left(c^{*} \lambda^{*}+\gamma_{2}\right)$ and

$$
\begin{aligned}
c^{*} \lambda^{*} & =M\left(\lambda^{*}\right) \\
& =d\left[e^{\lambda^{*} \cos \theta}+e^{-\lambda^{*} \cos \theta}+e^{\lambda^{*} \sin \theta}+e^{-\lambda^{*} \sin \theta}-4\right]+\left[f^{\prime}(0)-\gamma_{1}\right]+\frac{\gamma_{1} \gamma_{2}}{c^{*} \lambda^{*}+\gamma_{2}},
\end{aligned}
$$

that is, $\Delta\left(c^{*}, \lambda^{*}\right)=0$. Moreover, from (2.1) we obtain

$$
M(\lambda)=d\left[e^{\lambda \cos \theta}+e^{-\lambda \cos \theta}+e^{\lambda \sin \theta}+e^{-\lambda \sin \theta}-4\right]+\left[f^{\prime}(0)-\gamma_{1}\right]+\frac{\gamma_{1} \gamma_{2}}{M(\lambda)+\gamma_{2}} .
$$


Differentiating with respect to $\lambda$ this equality, we get

$$
\begin{aligned}
c^{*} & =M^{\prime}\left(\lambda^{*}\right) \\
& =d\left[\cos \theta e^{\lambda^{*} \cos \theta}-\cos \theta e^{-\lambda^{*} \cos \theta}+\sin \theta e^{\lambda^{*} \sin \theta}-\sin \theta e^{-\lambda^{*} \sin \theta}\right]-\frac{\gamma_{1} \gamma_{2} M^{\prime}\left(\lambda^{*}\right)}{\left[M\left(\lambda^{*}\right)+\gamma_{2}\right]^{2}} \\
& =d\left[\cos \theta e^{\lambda^{*} \cos \theta}-\cos \theta e^{-\lambda^{*} \cos \theta}+\sin \theta e^{\lambda^{*} \sin \theta}-\sin \theta e^{-\lambda^{*} \sin \theta}\right]-\frac{\gamma_{1} \gamma_{2} c^{*}}{\left[c^{*} \lambda^{*}+\gamma_{2}\right]^{2}},
\end{aligned}
$$

which implies that $\left.\frac{\partial}{\partial \lambda} \Delta\left(c^{*}, \lambda\right)\right|_{\lambda=\lambda^{*}}=0$. Hence $\left(c^{*}, \lambda^{*}\right)$ is also a solution of system (2.2). By the uniqueness of solutions of $(2.2)$ we deduce that $\left(c^{*}, \lambda^{*}\right)=\left(c_{*}, \lambda_{*}\right)$. This completes the proof.

By Lemmas 2.3-2.4, for each $c>c_{*}(\theta), c \lambda_{1}(c)=M\left(\lambda_{1}(c)\right)$, and $c \lambda>M(\lambda)$ for all $\lambda \in$ $\left(\lambda_{1}(c), \lambda_{*}\right)$. Let $v\left(\lambda_{1}\right)=\left(v_{1}\left(\lambda_{1}\right), v_{2}\left(\lambda_{1}\right)\right)$ be the eigenvector associated with $M\left(\lambda_{1}(c)\right)$.

\section{Stability of traveling wavefronts}

In this section, we consider the stability of the noncritical traveling wavefronts of (1.1) with given wave direction and speed. We always assume that $\Phi(\xi)=(\phi(\xi), \psi(\xi)), \xi=i \cos \theta+$ $j \sin \theta+c t$, is a traveling wavefront of (1.1) with speed $c>c_{*}(\theta)$ and direction $\theta \in[0,2 \pi]$ connecting $\mathbf{0}$ and $\mathbf{K}$. Without loss of generality, we extend $f$ satisfying $\left(A_{1}\right)$ and $\left(A_{2}\right)$ to $\hat{f}:[0, \infty) \rightarrow \mathbb{R}$ by

$$
\hat{f}_{j}(u):= \begin{cases}f_{j}(u), & u \in[0,1], \\ f_{j}^{\prime}(1)(u-1), & u \in[1,+\infty) .\end{cases}
$$

For convenience, we still denote $\hat{f}$ by $f$ in the rest of this section.

We first prove the following comparison theorem for a related initial-boundary problem of (1.1), which will play an important role in proving our main result. Given any $X_{0} \in$ $\mathbb{R} \cup\{-\infty\}$, define the domain $\Omega_{X_{0}}$ and its boundary $\partial \Omega_{X_{0}}$ as follows:

$$
\Omega_{X_{0}}:=\left\{(i, j, t) \in \mathbb{Z}^{2} \times \mathbb{R}_{+} \mid i \cos \theta+j \sin \theta+c t>X_{0}, t>0\right\}
$$

and

$$
\begin{gathered}
\partial \Omega_{X_{0}}:=\left\{(i, j, t) \in \mathbb{Z}^{2} \times \mathbb{R}_{+} \mid i \cos \theta+j \sin \theta+c t>X_{0},\right. \\
\left.t=0 \text { or } i \cos \theta+j \sin \theta+c t=X_{0}, t \geq 0\right\} .
\end{gathered}
$$

Lemma 3.1 Given any $\beta \in \mathbb{R}$, assume that $W_{i, j}^{ \pm}(t)=\left(u_{i, j}^{ \pm}(t), v_{i, j}^{ \pm}(t)\right): \mathbb{Z}^{2} \times \mathbb{R}_{+} \rightarrow \mathbb{R}^{2}$ are two functions satisfying

(i) $W_{i, j}^{+}(t) \geq \mathbf{0}$ and $W_{i, j}^{-}(t) \leq \mathbf{K}$ for all $(i, j, t) \in \mathbb{Z}^{2} \times \mathbb{R}^{+}$;

(ii) $W_{i, j}^{+}(t) \geq W_{i, j}^{-}(t)$ for all $(i, j, t) \in\left(\mathbb{Z}^{2} \times \mathbb{R}_{+}\right) \backslash \Omega_{X_{0}}$;

(iii) For $(i, j, t) \in \Omega_{X_{0}}$, we have

$$
\left\{\begin{array}{l}
\frac{\mathrm{d} u_{i, j}^{+}(t)}{\mathrm{d} t} \geq\left(\Delta_{2} u^{+}\right)_{i, j}+\beta u_{i, j}^{+}(t)+\gamma_{2} v_{i, j}^{+}(t), \\
\frac{\mathrm{d} v_{i, j}^{+}(t)}{\mathrm{d} t} \geq \gamma_{1} u_{i, j}^{+}(t)-\gamma_{2} v_{i, j}^{+}(t),
\end{array}\right.
$$


and

$$
\left\{\begin{array}{l}
\frac{\mathrm{d} u_{i, j}^{-}(t)}{\mathrm{d} t} \leq\left(\Delta_{2} u^{-}\right)_{i, j}+\beta u_{i, j}^{-}(t)+\gamma_{2} v_{i, j}^{-}(t), \\
\frac{\mathrm{d} v_{i, j}^{-}(t)}{\mathrm{d} t} \leq \gamma_{1} u_{i, j}^{-}(t)-\gamma_{2} v_{i, j}^{-}(t) .
\end{array}\right.
$$

Then $W_{i, j}^{+}(t) \geq W_{i, j}^{-}(t)$ for all $(i, j, t) \in \mathbb{Z}^{2} \times \mathbb{R}^{+}$.

Proof Take

$$
W_{i, j}(t)=\left(W_{i, j}^{(1)}(t), W_{i, j}^{(2)}(t)\right):=W_{i, j}^{+}(t)-W_{i, j}^{-}(t), \quad \forall(i, j) \in \mathbb{Z}^{2}, t \geq 0
$$

By our assumptions we see that $W_{i, j}(t)$ is bounded from below by $-\mathbf{K}$ and $W_{i, j}(t) \geq \mathbf{0}$ for all $(i, j, t) \in\left(\mathbb{Z}^{2} \times \mathbb{R}_{+}\right) \backslash \Omega_{X_{0}}$. Define the function

$$
\bar{W}(t)=\min _{l=1,2} \inf _{(i, j, t) \in \bar{\Omega}_{X_{0}}} W_{i, j}^{(l)}(t) \quad \text { for } t \geq 0 .
$$

It is clear that $\bar{W}(0) \geq 0$ and $\bar{W}(t)$ is a continuous function on $[0, \infty)$.

Fix $\delta>\max \left\{\beta+\gamma_{2}, \gamma_{1}\right\}$. Note that

$$
\liminf _{t \rightarrow \infty} \bar{W}(t) e^{-\delta t} \geq-\liminf _{t \rightarrow \infty} \min \{1, K\} e^{-\delta t}=0
$$

We claim that $\bar{W}(t) \geq 0$ for all $t \geq 0$. If this claim is not true, then there exists $t_{0}>0$ such that $\bar{W}\left(t_{0}\right)<0$ and

$$
\bar{W}\left(t_{0}\right) e^{-\delta t_{0}}=\min _{t \in\left[0, t_{0}\right]} \bar{W}(t) e^{-\delta t}<\bar{W}(s) e^{-\delta s}, \quad \forall s \in\left[0, t_{0}\right) .
$$

It is easy to see that there exist $l_{0} \in\{1,2\}$ and a sequence $\left\{\left(i_{k}, j_{k}\right)\right\}_{k=1}^{+\infty} \subseteq \mathbb{Z}^{2}$ such that

$$
W_{i_{k}, j_{k}}^{\left(l_{0}\right)}\left(t_{0}\right)<0, \quad \forall k \geq 1, \quad \text { and } \quad \lim _{k \rightarrow+\infty} W_{i_{k}, j_{k}}^{\left(l_{0}\right)}\left(t_{0}\right)=\bar{W}\left(t_{0}\right)
$$

Let $\left\{t_{k}\right\}_{k=1}^{+\infty}$ be a sequence in $\left(0, t_{0}\right]$ such that

$$
W_{i_{k}, j_{k}}^{\left(l_{0}\right)}\left(t_{k}\right) e^{-\delta t_{k}}=\min _{t \in\left[0, t_{0}\right]} W_{i_{k}, j_{k}}^{\left(l_{0}\right)}(t) e^{-\delta t}<0
$$

Since $W_{i, j}^{\left(l_{0}\right)}(t) \geq 0$ for any $(i, j, t) \in\left(\mathbb{Z}^{2} \times \mathbb{R}_{+}\right) \backslash \Omega_{X_{0}}$, we must have $\left(i_{k}, j_{k}, t_{k}\right) \in \Omega_{X_{0}}$. It then follows from (3.1) that $\lim _{k \rightarrow+\infty} t_{k}=t_{0}$. By the definition of $\bar{W}(t)$ we have

$$
\bar{W}\left(t_{k}\right) \leq W_{i_{k}, j_{k}}^{\left(l_{0}\right)}\left(t_{k}\right) \leq W_{i_{k}, j_{k}}^{\left(l_{0}\right)}\left(t_{0}\right) e^{-\delta\left(t_{0}-t_{k}\right)}
$$

which implies that $\lim _{k \rightarrow+\infty} W_{i_{k}, j_{k}}^{\left(l_{0}\right)}\left(t_{k}\right)=\bar{W}\left(t_{0}\right)<0$ by taking $k \rightarrow+\infty$.

We distinguish two cases.

Case 1. $l_{0}=1$. By the definition of $\bar{W}(t)$ and the fact $W_{i, j}^{\left(l_{0}\right)}(t) \geq 0$ for any $(i, j, t) \in\left(\mathbb{Z}^{2} \times\right.$ $\left.\mathbb{R}_{+}\right) \backslash \Omega_{X_{0}}$, for each $k \geq 1$, we obtain

$$
\begin{aligned}
\left(\Delta_{2} W^{(1)}\right)_{i_{k}, j_{k}}\left(t_{k}\right) & =d\left[W_{i_{k}+1, j_{k}}^{(1)}\left(t_{k}\right)+W_{i_{k}-1, j_{k}}^{(1)}\left(t_{k}\right)+W_{i_{k}, j_{k}+1}^{(1)}\left(t_{k}\right)+W_{i_{k}, j_{k}-1}^{(1)}\left(t_{k}\right)-4 W_{i_{k}, j_{k}}^{(1)}\left(t_{k}\right)\right] \\
& \geq 4 d \min \left\{0, \bar{W}\left(t_{k}\right)\right\}-4 d W_{i_{k}, j_{k}}^{(1)}\left(t_{k}\right) .
\end{aligned}
$$


Then by (3.2) we have

$$
\begin{aligned}
0 & \geq\left. e^{\delta t_{k}} \frac{\mathrm{d}}{\mathrm{d} t}\left(W_{i_{k}, j_{k}}^{(1)}(t) e^{-\delta t}\right)\right|_{t=t_{k}^{-}} \\
& =\frac{\mathrm{d}}{\mathrm{d} t} W_{i_{k}, j_{k}}^{(1)}\left(t_{k}\right)-\delta W_{i_{k}, j_{k}}^{(1)}\left(t_{k}\right) \\
& \geq\left(\Delta_{2} W^{(1)}\right)_{i_{k}, j_{k}}\left(t_{k}\right)+(\beta-\delta) W_{i_{k}, j_{k}}^{(1)}\left(t_{k}\right)+\gamma_{2} W_{i_{k}, j_{k}}^{(2)}\left(t_{k}\right) \\
& \geq 4 d \min \left\{0, \bar{W}\left(t_{k}\right)\right\}+(\beta-\delta-4 d) W_{i_{k}, j_{k}}^{(1)}\left(t_{k}\right)+\gamma_{2} \bar{W}\left(t_{k}\right) .
\end{aligned}
$$

Note that $\bar{W}\left(t_{0}\right)<0$ and $\delta>\beta+\gamma_{2}$. Sending $k \rightarrow \infty$, we get $0 \geq \bar{W}\left(t_{0}\right)\left[\beta+\gamma_{2}-\delta\right]>0$, a contradiction.

Case 2. $l_{0}=2$. Similarly, we have

$$
0 \geq \frac{\mathrm{d}}{\mathrm{d} t} W_{i_{k}, j_{k}}^{(2)}\left(t_{k}\right)-\delta W_{i_{k}, j_{k}}^{(2)}\left(t_{k}\right) \geq \gamma_{1} \bar{W}\left(t_{k}\right)+\left(-\gamma_{2}-\delta\right) W_{i_{k}, j_{k}}^{(2)}\left(t_{k}\right)
$$

Letting $k \rightarrow \infty$, we get $0 \geq \bar{W}\left(t_{0}\right)\left[\gamma_{1}-\gamma_{2}-\delta\right]>0$, a contradiction.

These contradictions show that $\bar{W}(t) \geq 0$ for all $t \geq 0$, and hence $W_{i, j}^{+}(t) \geq W_{i, j}^{-}(t)$ for all $(i, j, t) \in \mathbb{Z}^{2} \times \mathbb{R}^{+}$. This completes the proof.

Let $\lambda_{K}$ be the principle eigenvalue of the matrix

$$
\left(\begin{array}{cc}
f^{\prime}(1)-\gamma_{1} & \gamma_{2} \\
\gamma_{1} & -\gamma_{2}
\end{array}\right)
$$

and let $\varrho=\left(\varrho_{1}, \varrho_{2}\right) \gg(0,0)$ be the associated eigenvector. Thus

$$
\left\{\begin{array}{l}
\lambda_{K} \varrho_{1}=\left(f^{\prime}(1)-\gamma_{1}\right) \varrho_{1}+\gamma_{2} \varrho_{2}, \\
\lambda_{K} \varrho_{2}=\gamma_{1} \varrho_{1}-\gamma_{2} \varrho_{2} .
\end{array}\right.
$$

Since $f^{\prime}(1)<0$, it is easy to see that $\lambda_{K}<0$. In fact, suppose on the contrary that $\lambda_{K} \geq 0$. Then $\lambda_{K} \varrho_{2}=\gamma_{1} \varrho_{1}-\gamma_{2} \varrho_{2} \geq 0$, and hence

$$
\lambda_{K} \varrho_{1}=\left(f^{\prime}(1)-\gamma_{1}\right) \varrho_{1}+\gamma_{2} \varrho_{2} \leq f^{\prime}(1),
$$

which yields that $f^{\prime}(1) \geq \lambda_{K} \geq 0$. This contradicts the assumption that $f^{\prime}(1)<0$.

Take $\bar{\mu} \in\left(0,-\lambda_{K}\right)$ and $\epsilon \in\left(0, \min \left\{\bar{\mu}, \lambda_{*}(c)-\lambda_{1}(c)\right\}\right)$. Since $f \in C^{1}([0, \infty))$ and $f^{\prime}(u)=f^{\prime}(1)$ for all $u \geq 1$, there exists $\delta_{0} \in(0,1)$ such that

$$
f^{\prime}(u)<f^{\prime}(1)+\epsilon \quad \text { for any } u \geq 1-\delta_{0} .
$$

Noting that $\phi(+\infty)=1$, we can choose $M_{0}>0$ sufficiently large such that

$$
\phi(\xi) \in\left[1-\delta_{0}, 1\right) \quad \text { for all } \xi \geq M_{0}
$$


We now define the weighted function

$$
\omega^{\epsilon}(i, j):= \begin{cases}\mathrm{e}^{-\lambda_{\epsilon}\left(i \cos \theta+j \sin \theta-M_{0}\right)}, & i \cos \theta+j \sin \theta \leq M_{0} \\ 1, & i \cos \theta+j \sin \theta>M_{0}\end{cases}
$$

where $\lambda_{\epsilon}:=\lambda_{1}(c)+\epsilon$. Note that $c \lambda_{\epsilon}>M\left(\lambda_{\epsilon}\right)$. Take $w^{0}=\left\{w_{i, j}^{0}\right\}_{(i, j) \in \mathbb{Z}^{2}}=\left\{\left(U_{i, j}^{0}, V_{i, j}^{0}\right)\right\}_{(i, j) \in \mathbb{Z}^{2}}$. We now state the main result.

Theorem 3.2 Assume that $\mathbf{0} \leq w_{i, j}^{0} \leq \mathbf{K}$ and $\left\|w_{i, j}^{0}-\Phi(i \cos \theta+j \sin \theta)\right\| \omega^{\epsilon}(i, j)$ is bounded on $\mathbb{Z}^{2}$. Then there exists $\mu_{0}>0$ such that the unique solution $w_{i, j}\left(t ; w^{0}\right)=\left(u_{i, j}\left(t ; w^{0}\right)\right.$, $\left.v_{i, j}\left(t ; w^{0}\right)\right)$ of $(1.1)$ satisfies $\mathbf{0} \leq w_{i, j}\left(t ; w^{0}\right) \leq \mathbf{K}$ for $(i, j) \in \mathbb{Z}^{2}, t \geq 0$, and for some $C>0$,

$$
\sup _{(i, j) \in \mathbb{Z}^{2}}\left\|w_{i, j}\left(t ; w^{0}\right)-\Phi(i \cos \theta+j \sin \theta+c t)\right\| \leq C e^{-\mu_{0} t}, \quad \forall t \geq 0
$$

Proof Take $W_{i, j}^{ \pm, 0}=\left(U_{i, j}^{ \pm, 0}, V_{i, j}^{ \pm, 0}\right)$, where

$$
\begin{array}{ll}
U_{i, j}^{+, 0}:=\max \left\{u_{i, j}^{0}, \phi(i \cos \theta+j \sin \theta)\right\}, & \\
V_{i, j}^{+, 0}:=\max \left\{v_{i, j}^{0}, \psi(i \cos \theta+j \sin \theta)\right\}, \quad(i, j) \in \mathbb{Z}^{2}, \\
U_{i, j}^{-, 0}:=\min \left\{u_{i, j}^{0}, \phi(i \cos \theta+j \sin \theta)\right\}, \\
V_{i, j}^{-, 0}:=\min \left\{v_{i, j}^{0}, \psi(i \cos \theta+j \sin \theta)\right\}, \quad(i, j) \in \mathbb{Z}^{2} .
\end{array}
$$

Let $W_{i, j}^{ \pm}(t)=\left(U_{i, j}^{ \pm}(t), V_{i, j}^{ \pm}(t)\right)$ be the unique solution of (1.1) with the initial value $W^{ \pm, 0}=$ $\left\{W_{i, j}^{ \pm, 0}\right\}_{(i, j) \in \mathbb{Z}^{2}}$. By comparison theorem we obtain

$$
\mathbf{0} \leq W_{i, j}^{-}(t) \leq w_{i, j}\left(t ; w^{0}\right), \quad \Phi(i \cos \theta+j \sin \theta+c t) \leq W_{i, j}^{+}(t) \leq \mathbf{K}, \quad(i, j) \in \mathbb{Z}^{2}, t>0
$$

which implies that

$$
\begin{aligned}
& \left\|w_{i, j}\left(t ; w^{0}\right)-\Phi(i \cos \theta+j \sin \theta+c t)\right\| \\
& \quad \leq \max \left\{\left\|W_{i, j}^{+}(t)-\Phi(i \cos \theta+j \sin \theta+c t)\right\|,\left\|W_{i, j}^{-}(t)-\Phi(i \cos \theta+j \sin \theta+c t)\right\|\right\}
\end{aligned}
$$

for any $(i, j) \in \mathbb{Z}^{2}, t>0$. Thus, to prove the theorem, it suffices to prove the convergence of $W_{i, j}^{ \pm}(t)$ to $\Phi(i \cos \theta+j \sin \theta+c t)$. We only prove that $W_{i, j}^{+}(t)$ converges to $\Phi(i \cos \theta+j \sin \theta+$ $c t$ ) in time, since the other case can be treated similarly.

Denote

$$
Z_{i, j}(t)=\left(Z_{i, j}^{(1)}(t), Z_{i, j}^{(2)}(t)\right):=W_{i, j}^{+}(t)-\Phi(i \cos \theta+j \sin \theta+c t), \quad(i, j) \in \mathbb{Z}^{2}, t>0
$$

It is clear that, for all $(i, j) \in \mathbb{Z}^{2}$ and $t>0$,

$$
\begin{aligned}
& Z_{i, j}(t) \geq \mathbf{0}, \\
& 0 \leq Z_{i, j}^{(1)}(0) \leq\left|u_{i, j}^{0}-\phi(i \cos \theta+j \sin \theta)\right|, \\
& 0 \leq Z_{i, j}^{(2)}(0) \leq\left|v_{i, j}^{0}-\psi(i \cos \theta+j \sin \theta)\right| .
\end{aligned}
$$

Hence $\left\|Z_{i, j}(0)\right\| w^{\epsilon}(i, j)$ is bounded on $\mathbb{Z}^{2}$. 
We divide the remainder of the proof into two steps.

Step 1. We show that there exists $C_{1}>0$ such that

$$
Z_{i, j}(t) \leq C_{1} v\left(\lambda_{\epsilon}\right) \mathrm{e}^{-\left(c \lambda_{\epsilon}-M\left(\lambda_{\epsilon}\right)\right) t}, \quad \forall(i, j, t) \in\left(\mathbb{Z}^{2} \times \mathbb{R}^{+}\right) \backslash \Omega_{M_{0}} .
$$

By the assumption $f^{\prime}(u) \leq f^{\prime}(0), u \in[0,1]$, direction computation shows that

$$
\begin{aligned}
\frac{\mathrm{d} Z_{i, j}^{(1)}(t)}{\mathrm{d} t}= & d\left[Z_{i+1, j}^{(1)}+Z_{i-1, j}^{(1)}+Z_{i, j+1}^{(1)}+Z_{i, j-1}^{(1)}-4 Z_{i, j}^{(1)}\right]-\gamma_{1} Z_{i, j}^{(1)}(t)+\gamma_{2} Z_{i, j}^{(2)}(t) \\
& +f\left(U_{i, j}^{+}(t)\right)-f(\phi(i \cos \theta+j \sin \theta+c t)) \\
\leq & d\left[Z_{i+1, j}^{(1)}+Z_{i-1, j}^{(1)}+Z_{i, j+1}^{(1)}+Z_{i, j-1}^{(1)}-4 Z_{i, j}^{(1)}\right]+\left(f^{\prime}(0)-\gamma_{1}\right) Z_{i, j}^{(1)}(t)+\gamma_{2} Z_{i, j}(t)
\end{aligned}
$$

and

$$
\frac{\mathrm{d} Z_{i, j}^{(2)}(t)}{\mathrm{d} t}=\gamma_{1} Z_{i, j}^{(1)}(t)-\gamma_{2} Z_{i, j}^{(2)}(t)
$$

Let $v\left(\lambda_{\epsilon}\right)=\left(v_{1}\left(\lambda_{\epsilon}\right), v_{2}\left(\lambda_{\epsilon}\right)\right) \gg \mathbf{0}$ be the eigenvector of (2.1) corresponding to $M\left(\lambda_{\epsilon}\right)$. By the boundedness of $\left\|Z_{i, j}(0)\right\| w^{\epsilon}(i, j)$ on $\mathbb{Z}^{2}$ we can choose a sufficiently large $C_{1}>0$ such that

$$
Z_{i, j}(0) \leq C_{1} v\left(\lambda_{\epsilon}\right) \mathrm{e}^{\lambda_{\epsilon}\left(i \cos \theta+j \sin \theta-M_{0}\right)}, \quad i, j \in \mathbb{Z} .
$$

Define the function $\bar{Z}_{i, j}(t)=\left(\bar{Z}_{i, j}^{(1)}(t), \bar{Z}_{i, j}^{(2)}(t)\right)$ as follows:

$$
\bar{Z}_{i, j}(t)=C_{1} v\left(\lambda_{\epsilon}\right) \mathrm{e}^{\lambda_{\epsilon}\left(i \cos \theta+j \sin \theta-M_{0}\right)+M\left(\lambda_{\epsilon}\right) t}, \quad i, j \in \mathbb{Z}, t \geq 0 .
$$

Using (2.1), we can easily verify that

$$
\begin{aligned}
\frac{\mathrm{d} \bar{Z}_{i, j}^{(1)}(t)}{\mathrm{d} t}= & C_{1} M\left(\lambda_{\epsilon}\right) v_{1}\left(\lambda_{\epsilon}\right) \mathrm{e}^{\lambda_{\epsilon}\left(i \cos \theta+j \sin \theta-M_{0}\right)+M\left(\lambda_{\epsilon}\right) t} \\
= & C_{1} \mathrm{e}^{\lambda_{\epsilon}\left(i \cos \theta+j \sin \theta-M_{0}\right)+M\left(\lambda_{\epsilon}\right) t} \\
& \times\left\{d\left[e^{\lambda_{\epsilon} \cos \theta}+e^{-\lambda_{\epsilon} \cos \theta}+e^{\lambda_{\epsilon} \sin \theta}+e^{-\lambda_{\epsilon} \sin \theta}-4\right] v_{1}\left(\lambda_{\epsilon}\right)\right. \\
& \left.+\left[f^{\prime}(0)-\gamma_{1}\right] v_{1}\left(\lambda_{\epsilon}\right)+\gamma_{2} v_{2}\left(\lambda_{\epsilon}\right)\right\} \\
= & d\left[\bar{Z}_{i+1, j}^{(1)}+\bar{Z}_{i-1, j}^{(1)}+\bar{Z}_{i, j+1}^{(1)}+\bar{Z}_{i, j-1}^{(1)}-4 \bar{Z}_{i, j}^{(1)}\right]+\left(f^{\prime}(0)-\gamma_{1}\right) \bar{Z}_{i, j}^{(1)}(t)+\gamma_{2} \bar{Z}_{i, j}^{(2)}(t)
\end{aligned}
$$

for $i, j \in \mathbb{Z}, t>0$. Similarly, we have

$$
\frac{\mathrm{d} \bar{Z}_{i, j}^{(2)}(t)}{\mathrm{d} t}=\gamma_{1} \bar{Z}_{i, j}^{(1)}(t)-\gamma_{2} \bar{Z}_{i, j}^{(2)}(t), \quad i, j \in \mathbb{Z}, t>0
$$

Applying Lemma 3.1 with $X_{0}=-\infty$ and $\beta=f^{\prime}(0)-\gamma_{1}$, we get

$$
Z_{i, j}(t) \leq \bar{Z}_{i, j}(t), \quad \forall i, j \in \mathbb{Z}, t>0 .
$$


Then, for any $i, j \in \mathbb{Z}, t \geq 0$ with $i \cos \theta+j \sin \theta+c t \leq M_{0}$, we have

$$
\begin{aligned}
Z_{i, j}(t) & \leq C_{1} v\left(\lambda_{\epsilon}\right) \mathrm{e}^{\lambda_{\epsilon}\left(i \cos \theta+j \sin \theta-M_{0}\right)+M\left(\lambda_{\epsilon}\right) t} \\
& =C_{1} v\left(\lambda_{\epsilon}\right) \mathrm{e}^{\lambda_{\epsilon}\left(i \cos \theta+j \sin \theta+c t-M_{0}\right)} \mathrm{e}^{-\left(c \lambda_{\epsilon}-M\left(\lambda_{\epsilon}\right)\right) t} \leq C_{1} v\left(\lambda_{\epsilon}\right) \mathrm{e}^{-\left(c \lambda_{\epsilon}-M\left(\lambda_{\epsilon}\right)\right) t} .
\end{aligned}
$$

This proves (3.8).

Step 2. Let $\mu_{0}=\min \left\{c \lambda_{\epsilon}-M\left(\lambda_{\epsilon}\right),-\lambda_{K}-\epsilon\right\}>0$. We prove that there exists $L_{2}>0$ such that

$$
Z_{i, j}(t) \leq L_{2} \varrho \mathrm{e}^{-\mu_{0} t}, \quad \forall i, j \in \mathbb{Z}, t \geq 0
$$

Note that $\Phi(i \cos \theta+j \sin \theta+c t) \leq W_{i, j}^{+}(t) \leq \mathbf{K}$ for all $i, j \in \mathbb{Z}$ and $t \geq 0$ and $\phi(\xi) \in\left[1-\delta_{0}, 1\right)$ for all $\xi \geq M_{0}$. Thus it follows from (3.4) that for any $(i, j, t) \in \Omega_{M_{0}}$,

$$
\begin{aligned}
\frac{\mathrm{d} Z_{i, j}^{(1)}(t)}{\mathrm{d} t} \leq & d\left[Z_{i+1, j}^{(1)}+Z_{i-1, j}^{(1)}+Z_{i, j+1}^{(1)}+Z_{i, j-1}^{(1)}-4 Z_{i, j}^{(1)}\right] \\
& +\left(f^{\prime}(1)-\gamma_{1}+\epsilon\right) Z_{i, j}^{(1)}(t)+\gamma_{2} Z_{i, j}^{(2)}(t)
\end{aligned}
$$

and

$$
\frac{\mathrm{d} Z_{i, j}^{(2)}(t)}{\mathrm{d} t}=\gamma_{1} Z_{i, j}^{(1)}(t)-\gamma_{2} Z_{i, j}^{(2)}(t)
$$

We can choose $L_{2}>0$ such that $L_{2} \varrho \geq \max \left\{C_{1} v\left(\lambda_{\epsilon}\right), \mathbf{K}\right\}$. Define

$$
\hat{Z}_{i, j}(t)=\left(\hat{Z}_{i, j}^{(1)}(t), \hat{Z}_{i, j}^{(2)}(t)\right):=L_{2} \varrho e^{-\mu_{0} t}, \quad \forall i, j \in \mathbb{Z}, t \geq 0
$$

It is clear that $\hat{Z}_{i, j}(0) \geq \mathbf{K} \geq Z_{i, j}(0)$. Since $\mu_{0} \leq c \lambda_{\epsilon}-M\left(\lambda_{\epsilon}\right)$, from (3.8) we see that $Z_{i, j}(t) \leq$ $\hat{Z}_{i, j}(t)$ for all $(i, j, t) \in\left(\mathbb{Z}^{2} \times \mathbb{R}^{+}\right) \backslash \Omega_{M_{0}}$.

Due to $\mu_{0} \leq-\lambda_{K}-\epsilon \leq-\lambda_{K}$, we get

$$
\begin{aligned}
\frac{\mathrm{d} \hat{Z}_{i, j}^{(1)}(t)}{\mathrm{d} t}= & -\mu_{0} \hat{Z}_{i, j}^{(1)}(t) \geq\left(\lambda_{K}+\epsilon\right) \hat{Z}_{i, j}^{(1)}(t) \\
= & f^{\prime}(1) \hat{Z}_{i, j}^{(1)}(t)-\gamma_{1} \hat{Z}_{i, j}^{(1)}(t)+\gamma_{2} \hat{Z}_{i, j}^{(2)}(t)+\epsilon \hat{Z}_{i, j}^{(1)}(t) \\
\geq & d\left[Z_{i+1, j}^{(1)}+Z_{i-1, j}^{(1)}+Z_{i, j+1}^{(1)}+Z_{i, j-1}^{(1)}-4 Z_{i, j}^{(1)}\right] \\
& +\left(f^{\prime}(1)-\gamma_{1}+\epsilon\right) \hat{Z}_{i, j}^{(1)}(t)+\gamma_{2} \hat{Z}_{i, j}^{(2)}(t)
\end{aligned}
$$

and

$$
\frac{\mathrm{d} \hat{Z}_{i, j}^{(2)}(t)}{\mathrm{d} t}=-\mu_{0} \hat{Z}_{i, j}^{(2)}(t) \geq \lambda_{K} \hat{Z}_{i, j}^{(2)}(t)=\gamma_{1} \hat{Z}_{i, j}^{(1)}(t)-\gamma_{2} \hat{Z}_{i, j}^{(2)}(t)
$$

Using Lemma 3.1 with $X_{0}=M_{0}$ and $\beta=f^{\prime}(1)-\gamma_{1}+\epsilon$, we deduce that $Z_{i, j}(t) \leq \hat{Z}_{i, j}(t)$ for all $i, j \in \mathbb{Z}$ and $t \geq 0$, that is, (3.9) holds. Letting $\tilde{M}:=L_{2}\|\varrho\|$, we conclude that

$$
\left\|Z_{i, j}(t)\right\|=\left\|W_{i, j}^{+}(t)-\Phi(i \cos \theta+j \sin \theta+c t)\right\| \leq \tilde{M} \mathrm{e}^{-\mu_{0} t} .
$$

This completes the proof. 


\section{Discussions}

In the previous sections, we have studied the stability of the traveling wave solutions of the two-dimensional lattice differential system (1.1). Since (1.1) is a monotone system, our method is mainly based on the comparison principle. We would like to mention that in the recent years, there are some results on the stability of the traveling wave solutions for nonmonotone diffusion systems by using the weighted energy method; see, for example, Wu, Zhao, and Liu [18] and Lin et al. [8].

On the other hand, by taking into account the effect of time delay, it is natural to consider the following delayed lattice differential system:

$$
\left\{\begin{array}{l}
\frac{\mathrm{d} u_{i, j}(t)}{\mathrm{d} t}=\left(\Delta_{2} u\right)_{i, j}+f\left(u_{i, j}(t), u_{i, j}(t-\tau)\right)-\gamma_{1} u_{i, j}(t)+\gamma_{2} v_{i, j}(t) \\
\frac{\mathrm{d} v_{i, j}(t)}{\mathrm{d} t}=\gamma_{1} u_{i, j}(t)-\gamma_{2} v_{i, j}(t) .
\end{array}\right.
$$

For this delayed system, if the function $f(u, v)$ is not monotone with respect to $v$, then the comparison principle cannot be applied to consider the stability of traveling waves. An interesting problem is to study the stability of the traveling waves for such nonmonotone lattice differential systems such as (4.1).

\section{Acknowledgements}

The authors would like to thank the anonymous referees for their valuable comments and suggestions which have led to an improvement of the presentation.

\section{Funding}

This work is partially supported by the NSF of China (11501482) and the NSF of Shaanxi Province of China (2018JM1006)

Availability of data and materials

Not applicable.

\section{Competing interests}

The author declares that she has no competing interests.

\section{Authors' contributions}

The main idea of this paper was proposed by the author, and the author completed the final manuscript alone. All authors read and approved the final manuscript.

\section{Publisher's Note}

Springer Nature remains neutral with regard to jurisdictional claims in published maps and institutional affiliations.

Received: 29 April 2019 Accepted: 14 October 2019 Published online: 23 October 2019

\section{References}

1. Chang, C.-H.: The stability of traveling wave solutions for a diffusive competition system of three species. J. Math. Anal. Appl. 459, 564-576 (2018)

2. Chen, X., Guo, J.-S.: Existence and asymptotic stability of travelling waves of discrete quasilinear monostable equations. J. Differ. Equ. 184, 549-569 (2002)

3. Chow, S.-N.: Lattice dynamical systems. In: Macki, J.W., Zecca, P. (eds.) Dynamical Systems. Lecture Notes in Math., vol. 1822, pp. 1-102. Springer, Berlin (2003)

4. Hadeler, K.P., Lewis, M.A.: Spatial dynamics of the diffusive logistic equation with a sedentary compartment. Can. Appl. Math. Q. 10, 473-499 (2002)

5. Hsu, C.-H., Lin, J.-J., Yang, T.-S.: Stability for monostable wave fronts of delayed lattice differential equations. J. Dyn. Differ. Equ. 29, 323-342 (2017)

6. Liu, X.-L., Pan, S.: Spreading speed in a nonmonotone equation with dispersal and delay. Mathematics 7, 291, 1-9 (2019)

7. Liang, X., Zhao, X.-Q.: Asymptotic speeds of spread and traveling waves for monotone semiflows with applications. Commun. Pure Appl. Math. 60, 1-40 (2007); Erratum: 61, 137-138 (2008)

8. Lin, C.-K., Lin, C.-T., Lin, Y., Mei, M.: Exponential stability of nonmonotone traveling waves for Nicholson's blowflies equation. SIAM J. Math. Anal. 46, 1053-1084 (2014)

9. Lin, C.-K., Mei, M.: On travelling wavefronts of the Nicholson's blowies equation with diffusion. Proc. R. Soc. Edinb. A $140,135-152(2010)$ 
10. Lin, G., Li, W.-T., Pan, S.: Travelling wavefronts in delayed lattice dynamical systems with global interaction. J. Differ. Equ. Appl. 16, 1429-1446 (2010)

11. Mei, M., Lin, C.-K., Lin, C.-T., So, J.W.H.: Traveling wavefronts for time-delayed reaction-diffusion equation: II nonlocal nonlinearity. J. Differ. Equ. 247, 511-529 (2009)

12. Mei, M., So, J.W.-H., Li, M., Shen, S.: Asymptotic stability of traveling waves for Nicholson's blowflies equation with diffusion. Proc. R. Soc. Edinb. A 134, 579-594 (2004)

13. Ouyang, Z., Ou, C.: Global stability and convergence rate of traveling waves for a nonlocal model in periodic media. Discrete Contin. Dyn. Syst., Ser. B 17, 993-1007 (2012)

14. Shorrocks, B., Swingland, I.R.: Living in a Patch Environment. Oxford University Press, New York (1990)

15. Smith, H.L.: Monotone Dynamical Systems: An Introduction to the Theory of Competitive and Cooperative Systems. Math. Surveys Monogr., vol. 41. Am. Math. Soc., Providence (1995)

16. Wang, X.-S., Zhao, X.-Q.: Pulsating waves of a partially degenerate reaction-diffusion system in a periodic habitat. J. Differ. Equ. 259, 7238-7259 (2015)

17. Wang, Z.-C., Li, W.-T., Ruan, S.: Existence and stability of traveling wave fronts in reaction advection diffusion equations with nonlocal delay. J. Differ. Equ. 238, 153-200 (2007)

18. Wu, S.-L., Zhao, H.-Q., Liu, S.: Asymptotic stability of traveling waves for delayed reaction-diffusion equations with crossing-monostability. Z. Angew. Math. Phys. 62, 377-397 (2011)

19. $W u, Y$., Xing, X.: Stability of traveling waves with critical speeds for $p$-degree Fisher-type equations. Discrete Contin. Dyn. Syst. 20, 1123-1139 (2008)

20. Zhang, K., Zhao, X.Q.: Asymptotic behaviour of a reaction-diffusion model with a quiescent stage. Proc. R. Soc. Lond. 463A, 1029-1043 (2007)

21. Zhang, P., Li, W.-T.: Monotonicity and uniqueness of traveling waves for a reaction-diffusion model with a quiescent stage. Nonlinear Anal. TMA 72, 2178-2189 (2010)

22. Zhao, H.-Q., Wu, S.-L.: Wave propagation for a reaction-diffusion model with a quiescent stage on a 2D spatial lattice. Nonlinear Anal., Real World Appl. 12, 1178-1191 (2011)

\section{Submit your manuscript to a SpringerOpen ${ }^{\circ}$ journal and benefit from:}

- Convenient online submission

- Rigorous peer review

- Open access: articles freely available online

- High visibility within the field

- Retaining the copyright to your article

Submit your next manuscript at $\boldsymbol{\nabla}$ springeropen.com 